\title{
Interactions between Maraviroc and the ABCB1, ABCG2, and ABCC2 Transporters: An Important Role in Transplacental Pharmacokinetics
}

\author{
Lenka Tupova, Martina Ceckova, Csilla Ambrus, Ales Sorf, Zuzana Ptackova, Zsuzsanna Gaborik, \\ and Frantisek Staud
}

Department of Pharmacology and Toxicology, Faculty of Pharmacy in Hradec Kralove, Charles University, Hradec Kralove, Czech Republic (L.T., M.C., A.S., Z.P., F.S.); Solvo Biotechnology, Budapest, Hungary (C.A., Z.G.)

Received April 23, 2019; accepted June 10, 2019

\begin{abstract}
Maraviroc is a chemokine receptor 5 (CCR5) inhibitor used in the treatment of human immunodeficiency virus (HIV) that also shows therapeutic potential for several autoimmune, cancer, and inflammatory diseases that can afflict pregnant women. However, only limited information exists on the mechanisms underlying the transplacental transfer of the drug. We aimed to expand the current knowledge base on how maraviroc interacts with several placental ATP-binding cassette (ABC) efflux transporters that have a recognized role in the protection of a developing fetus: $\mathrm{P}$-glycoprotein (ABCB1), breast cancer resistance protein (ABCG2), and multidrug resistance protein 2 (ABCC2). We found that maraviroc does not inhibit any of the three studied $A B C$ transporters and that its permeability is not affected by ABCG2 or ABCC2. However, our in vitro results revealed that maraviroc shows affinity for human ABCB1 and the endogenous canine P-glycoprotein (Abcb1) expressed in Madin-Darby canine kidney II (MDCKII) cells. Perfusion of rat term placenta showed accelerated transport of maraviroc in the fetal-tomaternal direction, which suggests that $A B C B 1 / A b c b 1$ facilitates in situ maraviroc transport. This transplacental transport was saturable and significantly diminished after the addition of the ABCB1/Abcb1
\end{abstract}

inhibitors elacridar, zosuquidar, and ritonavir. Our results indicate that neither $A B C G 2$ nor $A B C C 2$ influence maraviroc pharmacokinetic but that $A B C B 1 / A b c b 1$ may be partly responsible for the decreased transplacental permeability of maraviroc to the fetus. The strong affinity of maraviroc to Abcb1 found in our animal models necessitates studies in human tissue so that maraviroc pharmacokinetics in pregnant women can be fully understood.

\section{SIGNIFICANCE STATEMENT}

Antiretroviral drug maraviroc shows low toxicity and is thus a good candidate for prevention of mother-to-child transmission of human immunodeficiency virus when failure of recommended therapy occurs. Using in vitro cell-based experiments and in situ dually perfused rat term placenta, we examined maraviroc interaction with the placental $A B C$ drug transporters $A B C B 1, A B C G 2$, and $A B C C 2$. We demonstrate for the first time that placental $A B C B 1$ significantly reduces mother-to-fetus transport of maraviroc, which suggests that ABCB1 may be responsible for the low cord-blood/maternalblood ratio observed in humans.

\section{Introduction}

Maraviroc is a specific chemokine receptor 5 (CCR5) antagonist approved in 2007 for the treatment of adults with CCR5-tropic human immunodeficiency virus type $1(\mathrm{HIV}-1)$ infection and prior antiretroviral therapy ((OARAC, 2019a)). Due to its low toxicity, minimal side effects, and optimal pharmacotherapeutic profile, maraviroc was approved in 2016 for administration in children older than 2 years ((OARAC, 2019b)). Even though some clinical reports suggest that maraviroc is safe for the

The study was supported by the Grant Agency of the Charles University (GAUK 616216/C/2016 and SVV/2017/260-414); the Czech Science Foundation (GACR 17-16169S); and project EFSA-CDN (No. CZ.02.1.01/0.0/0.0/16_019/0000841) cofunded by ERDF. The funders had no role in study design, data collection and analysis, the decision to publish, or the preparation of the manuscript.

https://doi.org/10.1124/dmd.119.087684 fetus, maraviroc-based therapeutic regimens are currently not recommended for the treatment of pregnant women or prevention of motherto-child transmission of HIV due to limited data in pregnancy. However, the administration of this drug is not contraindicated in women who have experienced therapy failure or used and tolerated maraviroc before pregnancy ((OARAC, 2018)). Besides HIV infection, maraviroc has also shown beneficial therapeutic effects in several autoimmune and inflammatory diseases that are characterized by the dysregulation of CCR5 physiologic function (Woollard and Kanmogne, 2015). Moreover, experiments using breast and colorectal cancer models provide promising evidence that maraviroc may be able to slow down the progression of solid tumors in which CCR5 expression is associated with metastatic behavior (Pervaiz et al., 2015, 2019).

Maraviroc is administered orally with limited bioavailability (up to $23 \%$ ) due to metabolism via cytochrome P450 isoforms 3A4 and 3A5

ABBREVIATIONS: A-B, apical to basolateral; ABC, ATP-binding cassette; Abcb1, endogenous canine P-glycoprotein; ABCB1, P-glycoprotein; $A B C C 2$, multidrug resistance protein 2; ABCG2, breast cancer resistance protein; B-A, basolateral to apical; CCR5, chemokine receptor 5; DMEM, Dulbecco's modified Eagle's medium; ER, efflux ratio; F $\rightarrow$ M, fetal-to-maternal direction; GF120918, elacridar; $\left[{ }^{3} \mathrm{H}\right]-\mathrm{MVC}$, radiolabeled maraviroc; Hoechst 33342, 2-(4-ethoxyphenyl)-6-[6-(4-methylpiperazin-1-yl)-1H-benzimidazol-2-yl]-1H-benzimidazole; HIV, human immunodeficiency virus; KO, knockout; Ko143, tert-butyl 3-[(2S,5S,8S)-14-methoxy-2-(2-methylpropyl)-4,7-dioxo-3,6,17-triazatetracyclo[8.7.0.03,8.011,16]heptadeca1(10),11,13,15-tetraen-5-yl]propanoate; LY335979, zosuquidar; MDCKII cells, Madin-Darby canine kidney II cells; M $\rightarrow F$, maternal-to-fetal direction; MK-571, 3-[[3-[(E)-2-(7-chloroquinolin-2-yl)ethenyl]phenyl]-[3-(dimethylamino)-3-oxopropyl]sulfanylmethyl]sulfanylpropanoic acid; $\mathrm{R}_{\mathrm{r}}$, relative ratio. 
(CYP3A4 and CYP3A5) (Abel et al., 2008a,b; Lu et al., 2012). Additionally, experiments in Caco-2 cells found it to be a substrate of human P-glycoprotein (ABCB1), and maraviroc bioavailability was clearly increased in endogenous canine P-glycoprotein (Abcbla/b) knockout (KO) mice (Walker et al., 2005; Abel et al., 2008a,b). Therefore, ABCB1 could partly contribute to reduced drug bioavailability in humans after oral administration. ABCB1, an ATP-binding cassette $(\mathrm{ABC})$ drug efflux transporter, is considered to exert a protective role in the placental barrier by blocking the penetration of potentially toxic compounds from maternal blood to the fetus (Ceckova-Novotna et al., 2006; Han et al., 2018).

Breast cancer resistance protein (ABCG2) and multidrug resistancerelated protein type 2 (ABCC2) are additional members of the placental barrier that have fetus protective roles (Hahnova-Cygalova et al., 2011; Staud et al., 2012; Han et al., 2018). However, only limited data exist as to how maraviroc interacts with any of the aforementioned transporters. A low transplacental passage of maraviroc, calculated as cord blood/ maternal blood ratio (value of 0.33 ), was found after delivery in women treated with maraviroc during pregnancy (Calcagno et al., 2013; Colbers et al., 2015), indicating the possible involvement of ABC transporters or other efflux mechanisms. In this study, we addressed the possible interactions of maraviroc with ABCB1, ABCG2, and ABCC2 as well as evaluated how these transporters influence the transcellular passage of maraviroc using $\mathrm{ABC}$ transporter-overexpressing cells and dually perfused rat term placenta.

\section{Material and Methods}

Chemicals and Reagents. Radiolabeled maraviroc $\left(\left[{ }^{3} \mathrm{H}\right]-\mathrm{MVC}\right)$ was purchased from Moravek Biochemicals (Brea, CA). Dulbecco's modified Eagle's medium (DMEM), fetal bovine serum (FBS), sodium dodecyl sulfate (SDS), dimethyl sulfoxide (DMSO), 2-(4-ethoxyphenyl)-6-[6-(4-methylpiperazin-1-yl)$1 H$-benzimidazol-2-yl]- $1 H$-benzimidazole (Hoechst 33342), calcein-AM, dextran, and scintillation cocktail were all purchased from Sigma-Aldrich (St. Louis, MO). Gibco Opti-MEM was purchased from ThermoFisher Scientific (Waltham, MA). The antiretroviral drugs maraviroc and ritonavir were provided by the U.S. National Institutes of Health AIDS Reagent Program (Germantown, MD) or were purchased from MedChemExpress (Monmouth Junction, NJ). The dual ABCG2 and ABCB1 inhibitor GF120918 (elacridar), the specific ABCB1 inhibitor LY335979 (zosuquidar), and the specific $\mathrm{ABCG} 2$ inhibitor fumitremorgin $\mathrm{C}$ were purchased from Sigma-Aldrich. Pentobarbital (Nembutal) was obtained from Abbott Laboratories (Abbott Park, IL).

Cell Cultures. Madin-Darby canine kidney II (MDCKII) cell lines, parental and those overexpressing the human $\mathrm{ABCB} 1, \mathrm{ABCG} 2$, or $\mathrm{ABCC} 2$ transporter, were obtained from Dr. A. Schinkel (Netherlands Cancer Institute, Amsterdam, the Netherlands). A MDCKII-Abcb1 biallelic KO cell clone was generated by GenScript (Leiden, the Netherlands) using the Clustered Regularly-Interspaced Short Palindromic Repeats (CRISPR/Cas9) system to knock out the canine Abcb1 gene, after which it was characterized by SOLVO Biotechnology Laboratories (Budapest, Hungary).

The genotype of this KO clone was determined by Sanger sequencing. The phenotype was verified through transport assays using the ABCB1/Abcb1specific substrate digoxin. Human epidermal cell lines the control cell line A431 and its $\mathrm{ABCB} 1$-overexpressing resistant variant $\mathrm{A} 431-\mathrm{ABCB} 1$ were kindly provided by Dr. Balázs Sarkadi (Hungarian Academy of Sciences, Budapest, Hungary).

All cells were cultured in DMEM complete high-glucose medium with L-glutamine, supplemented with $10 \%$ FBS, routinely cultivated in antibiotic-free medium at $37^{\circ} \mathrm{C}$ and $5 \% \mathrm{CO}_{2}$, and periodically tested for Mycoplasma contamination. Cells from passages 5 to 30 were used in the experiments. The DMSO concentrations did not exceed $0.5 \%$ in any of the experiments.

Animals. Pregnant Wistar rats were purchased from Velaz s.r.o. (Prague, Czech Republic) and maintained at 12-hour light/dark standard conditions with water and pellets ad libitum. The experiments were performed on day 21 of gestation. Fasted rats were anesthetized using pentobarbital administered into the tail vein at a dose of $40 \mathrm{mg} / \mathrm{kg}$. All the performed experiments were approved by the ethics committee of the Faculty of Pharmacy in Hradec Kralove (Charles University, Hradec Kralove, Czech Republic) and were performed in accordance with the U.S. National Institutes of Health Guide for the Care and Use of Laboratory Animals (1996) and the European Convention for the Protection of Vertebrate Animals Used for Experimental and Other Scientific Purposes.

Accumulation Assays in MDCKII ABCB1, ABCG2, or ABCC2 Cell Lines. MDCKII-ABCB1, MDCKII-ABCG2, and MDCKII-ABCC2 cells were seeded on 96-well plates at a density of $5 \times 10^{4}$ cells per well and incubated in DMEM for 24 hours to reach full confluence. On the day of the experiment, the culture medium was removed, and the cells were rinsed with warm PBS. The cells were preincubated for 15 minutes either in pure Opti-MEM or in the presence of a range of maraviroc concentrations $(1-50 \mu \mathrm{M})$ or one of the control transporter inhibitors: zosuquidar $(2 \mu \mathrm{M})$; tert-butyl 3-[(2S,5S,8S)-14-methoxy-2-(2-methylpropyl)4,7-dioxo-3,6,17-triazatetracyclo[8.7.0.03,8.011,16]heptadeca-1(10),11,13,15-tetraen-5yl]propanoate (Ko143, $2 \mu \mathrm{M})$; elacridar (2 $\mu \mathrm{M})$; or 3-[[3-[(E)-2-(7-chloroquinolin-2-yl) ethenyl]phenyl]-[3-(dimethylamino)-3-oxopropyl]sulfanylmethyl]sulfanylpropanoic acid (MK-571, $20 \mu \mathrm{M})$. A specific ABCB1 and ABCG2 fluorescent substrate Hoechst $33342(5 \mu \mathrm{g} / \mathrm{ml} / 8.9 \mu \mathrm{M})$ or a $\mathrm{ABCC} 2$ fluorescent substrate calcein-AM $(10 \mu \mathrm{M})$ was then added. Relative fluorescent units were measured immediately after the addition of Hoechst 33342 or calcein-AM in 1-minute intervals for 30 minutes at a constant temperature of $37^{\circ} \mathrm{C}$ using a microplate reader (Genios Plus; Tecan, Salzburg, Austria). The same experimental setup also was used for the MDCKII parental cells (data not shown).

Bidirectional Transport Experiments across Monolayers of MDCKII Parental, ABCB1, ABCG2, ABCC2, and Abcb1 KO Cell Lines. Transport assays were performed on $3-\mu \mathrm{M}$ microporous polycarbonate membrane filters (Transwell 3414; Costar Corning, New York, NY) with the parental cell lines and the MDCKII cell lines overexpressing ABCB1, ABCG2, or ABCC2, as described elsewhere (Neumanova et al., 2014). On the day of the experiment, the culture medium was removed, after which the monolayers were rinsed with PBS (at $37^{\circ} \mathrm{C}$ ) and then preincubated in Opti-MEM with or without the control inhibitor zosuquidar $(2 \mu \mathrm{M})$ for 15 minutes. The transport assay was initiated by the addition of $\left[{ }^{3} \mathrm{H}\right]-\mathrm{MVC} 0.07 \mu \mathrm{Ci} / \mathrm{ml}(23 \mathrm{nM})$ into the apical or the basal compartment. Samples of $100 \mu$ were collected at 2, 4, and 6 hours from the acceptor compartment, and radioactivity was measured by liquid scintillation counting (Tri-Carb 2900 TR; PerkinElmer, Waltham, MA). The concentration at each sampling point was expressed as a percentage and related to the stock solution. The efflux ratio $(E R=B-A / A-B)$ was calculated after a 2-hour incubation using the permeability coefficients $\left(\mathrm{P}_{\text {app }}\right)$. Fluorescein-5-isothiocyanate-dextran leakage was also analyzed after the experiments had finished, with rates lower than $1 \%$ per hour considered acceptable.

Transport assays in MDCKII-Abcb1 KO cells were performed at SOLVO Biotechnology Laboratories according to the same protocol described previously. To guarantee consistent experimental conditions, the maraviroc transport assays in MDCKII-parental cells were repeated at SOLVO, and the results did not significantly differ from those obtained from the experiments performed at the Charles University laboratories.

Accumulation Assay in Human Epidermoid A431 Cell Lines. A431 and A431-ABCB1 cells were seeded into 24-well plates (Techno Plastic Products, Trasadingen, Switzerland) at a density of $3 \times 10^{5}$ cells per well and cultured for 24 hours at $37^{\circ} \mathrm{C}$ and $5 \% \mathrm{CO}_{2}$. The culture medium was then removed, and the cells were washed in $\mathrm{PBS}\left(\right.$ at $\left.37^{\circ} \mathrm{C}\right)$. The experiment was initiated by an addition of $\left[{ }^{3} \mathrm{H}\right]$-maraviroc $0.2 \mu \mathrm{Ci} / \mathrm{ml}(65 \mathrm{nM})$ dissolved in Opti-MEM. Elacridar $(2 \mu \mathrm{M})$ - which served as a control ABCB 1 inhibitor-was added to the cells 15 minutes before the start of the experiment. Accumulation was stopped after 1 hour by removing the working solution and immediately washing the cells with ice-cold PBS $\left(4^{\circ} \mathrm{C}\right)$ twice. The cells were then lysed in $0.02 \%$ SDS, and maraviroc accumulation was detected by liquid scintillation counting and normalized to total protein content.

Dual Perfusion of Rat Term Placenta. The dual perfusion of rat term placenta was performed as described elsewhere (Staud et al., 2006; Neumanova et al., 2014). Briefly, pregnant rats were anesthetized on gestational day 21. One uterine horn was excised, and a suitable placenta was chosen. Maternal circulation was established by cannulation and perfusion of a uterine artery proximal to the selected placenta. The uterine vein was ligated behind the perfused placenta and cut so that the maternal solution could exit the circulation. Next, fetal compartment circulation was set up by cannulation of umbilical vessels between 

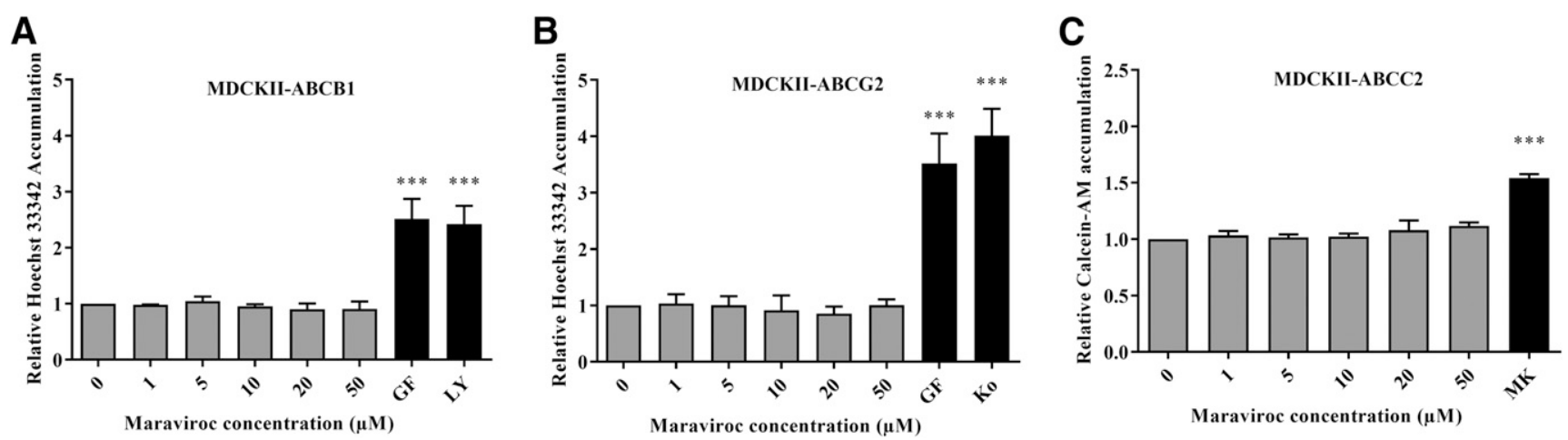

Fig. 1. The effect of maraviroc on Hoechst 33342 accumulation in MDCKII-ABCB1 (A) and MDCKII-ABCG2 (B) cells, and on calcein-AM accumulation in MDCKII-ABCC2 (C) cells after a 30-minute incubation. Control inhibitors: $2 \mu \mathrm{M}$ elacridar (GF), $2 \mu \mathrm{M}$ zosuquidar (LY), $2 \mu \mathrm{M}$ Ko143 (Ko), and $20 \mu \mathrm{M}$ MK-571 (MK). MDCKII parental cells were used to verify experiment validity (data not shown). Columns represent mean \pm S.D. from at least three independent experiments. Data obtained for model inhibitors and maraviroc were compared with untreated controls using one-way ANOVA, with Dunnett's multiple comparison test, $* * P \leq 0.01 ; * * * P \leq 0.001$.

the fetus and the selected placenta with collection of the outflow from the umbilical vein.

Two variations of the perfusion experiment were performed. In the first, an open-circuit perfusion setup was established to study the fetal-to-maternal $(\mathrm{F} \rightarrow \mathrm{M})$ and the maternal-to-fetal $(\mathrm{M} \rightarrow \mathrm{F})$ clearance of maraviroc. Radiolabeled $\left[{ }^{3} \mathrm{H}\right]$ MVC $(23 \mathrm{nM})$ was added to either the maternal or the fetal reservoir immediately after successful cannulation. Samples of fetal effluent were collected after a 5-minute stabilization period in 5-minute intervals into the preweighted tubes, ending at the 35th minute. Maraviroc concentrations were measured using liquid scintillation counting on Tri-Carb 2900 TR (Perkin Elmer) while transplacental clearance was calculated as described elsewhere (Staud et al., 2006).

In the second, a closed-circuit (recirculation) perfusion setup was established to study how placental transporters are involved in the transplacental passage of maraviroc. Both the maternal and the fetal sides were perfused with an equal amount of $\left[{ }^{3} \mathrm{H}\right]$-MVC $(23 \mathrm{nM})$, with or without the inhibitor. After a short 5-minute stabilization period, the fetal perfusate $(10 \mathrm{ml})$ was recirculated for 60 minutes. Samples $(100 \mu \mathrm{l})$ were collected every 10 minutes in duplicates from the maternal and the fetal reservoir, and maraviroc concentrations were measured using liquid scintillation counting. A high concentration of cold maraviroc $(100 \mu \mathrm{M})$ was used to determine the saturability of the transport. A specific ABCB1 inhibitor zosuquidar $(2 \mu \mathrm{M})$, a dual $\mathrm{ABCB} 1$ and $\mathrm{ABCG} 2$ inhibitor elacridar $(2 \mu \mathrm{M})$, a specific ABCG2 inhibitor fumitremorgin $\mathrm{C}(2 \mu \mathrm{M})$, and a nonspecific $\mathrm{ABCB} 1$ inhibitor ritonavir $(10 \mu \mathrm{M})$ were added to both sides to help identify the mechanism responsible for the active transport of maraviroc.

Statistical Analysis. The obtained data are presented as the mean \pm S.D. of at least three independent experiments. Statistical analysis was performed using GraphPad Prism software version 8.01 (GraphPad Software, La Jolla, CA). The $P$ values were calculated by one-way ANOVA or a nonparametric Mann-Whitney $U$-test with the threshold for statistical significance set at $P \leq 0.05$.

\section{Results}

Maraviroc Does Not Inhibit Human ABCB1, ABCG2, or ABCC2. The inhibitory potential of maraviroc on the $A B C$ efflux transporters was evaluated using accumulation assays with model transporter substrates. Maraviroc did not alter the Hoechst 33342 accumulation in the MDCKII-ABCB1 and MDCKII-ABCG2 cell lines, or the calcein$\mathrm{AM}$ accumulation in the MDCKII-ABCC 2 cells, even when applied at the highest concentration available based on its solubility (Fig. 1).

Maraviroc Is Not a Substrate of Human ABCG2 or ABCC2, but Shows Significant Transport by Canine Abcb1. Maraviroc transport across a monolayer of MDCKII cells was accelerated in the basolateralto-apical (B-A) direction in all the tested cell lines that overexpressed human efflux transporters. The ER ratio across the MDCKII-ABCB1 monolayer was 6.01 (Fig. 2A), while the respective ER ratios of maraviroc transport across MDCKII-ABCG2 and MDCKII-ABCC2 cell monolayers reached 8.33 and 5.10, respectively (data not shown). Interestingly, a similar asymmetry in the B-A direction was observed $(E R=5.01)$ for the transport of maraviroc in the MDCKII parental cell line (Fig. 2E). This imbalance was completely abolished in all of the tested cell lines after the addition of the ABCB1-specific inhibitor zosuquidar $(2 \mu \mathrm{M})$ (Fig. 2, B-D and F); this suggests that the endogenous canine Abcb1 was involved in the imbalanced B-A maraviroc transport observed in human transporteroverexpressing cell lines.

No asymmetry between maraviroc transport in the A-B or B-A directions was detected in canine Abcb1 KO MDCKII parental cells (Fig. 2G), which confirms that maraviroc has a strong affinity for endogenous Abcb1 in MDCKII cell lines. Similar transport behavior was also confirmed with the control Abcb1/ABCB1 substrate digoxin (Fig. 2H).

Maraviroc Is a Substrate of Human ABCB1. To test whether maraviroc is also transported by human $\mathrm{ABCB} 1$, we evaluated the accumulation of $\left[{ }^{3} \mathrm{H}\right]-\mathrm{MVC}$ in human epidermal carcinoma A431 cells, as well as how a model ABCB1 inhibitor affects this process. There was a 4.25-fold decrease in $\left[{ }^{3} \mathrm{H}\right]-\mathrm{MVC}$ accumulation in the ABCB1expressing cells relative to the control A431 cells (Fig. 3). The ABCB1 inhibitor elacridar $(2 \mu \mathrm{M})$ was able to partially reverse this effect, as maraviroc accumulation increased 1.92-fold after elacridar treatment. These results confirm that human ABCB1 is involved in the transport of maraviroc.

Dually Perfused Rat Term Placenta: Open Circuit System. When the maternal or the fetal side of the rat placenta was perfused by maraviroc $(23 \mathrm{nM})$, maraviroc transport in the $\mathrm{F} \rightarrow \mathrm{M}$ direction significantly accelerated (4.18-fold) relative to transport in the $\mathrm{M} \rightarrow \mathrm{F}$ direction (Fig. 4). This suggests that an active transport mechanism is involved in the transplacental passage of maraviroc in the $\mathrm{F} \rightarrow \mathrm{M}$ direction. Less than $2 \%$ of the maraviroc dose was retained in the placenta, indicating limited tissue binding.

Dually Perfused Rat Term Placenta: Closed Circuit System. Maraviroc Is a Substrate of Rat Placental Abcb1. To identify transporters involved in the transplacental passage of maraviroc, both sides of the placenta were infused with equal amounts of $\left[{ }^{3} \mathrm{H}\right]-\mathrm{MVC}$ (23 nM), with or without the inhibitor (Fig. 5). We found that maraviroc concentrations significantly decreased in the fetal compartment, confirming that the compound is actively transported against the concentration gradient in the $\mathrm{F} \rightarrow \mathrm{M}$ direction. This transport became saturated at a higher maraviroc concentration $(100 \mu \mathrm{M})$ and was significantly inhibited after the addition of the ABCB1 inhibitors elacridar $(2 \mu \mathrm{M})$, zosuquidar $(2 \mu \mathrm{M})$, and ritonavir 
A

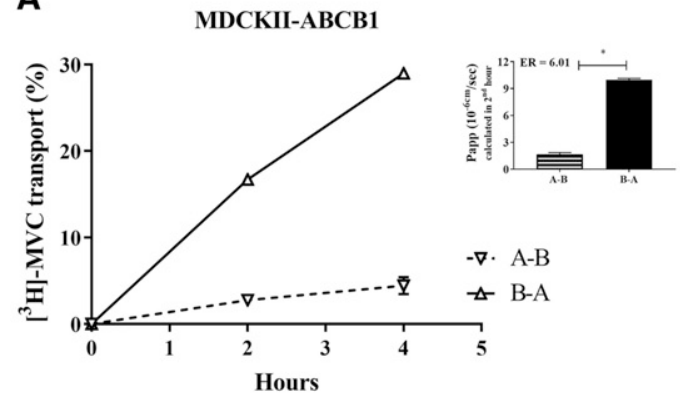

c

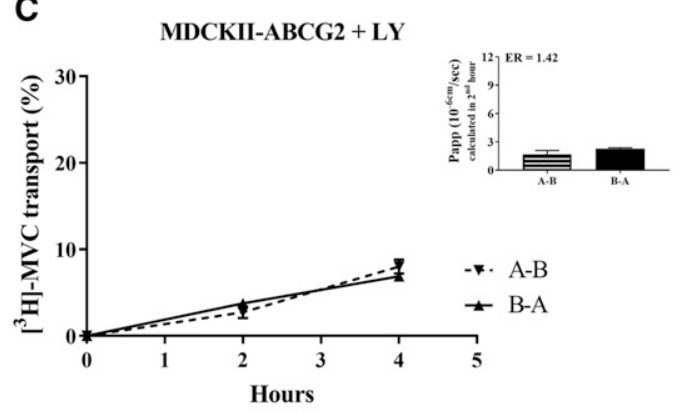

E

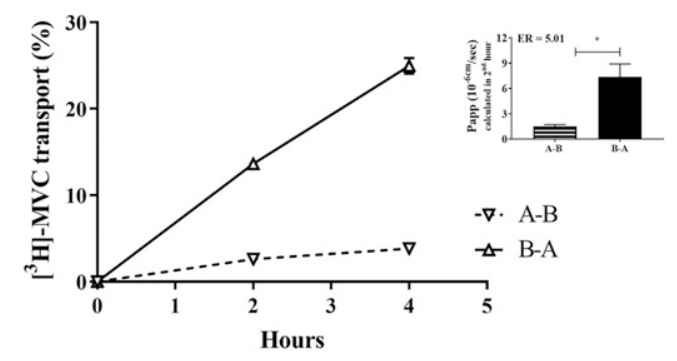

G

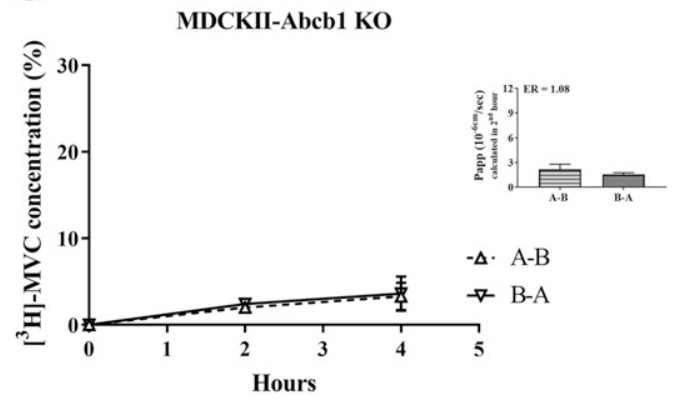

B

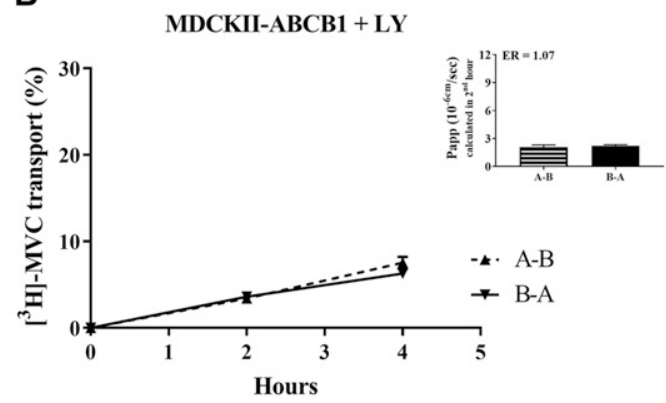

D

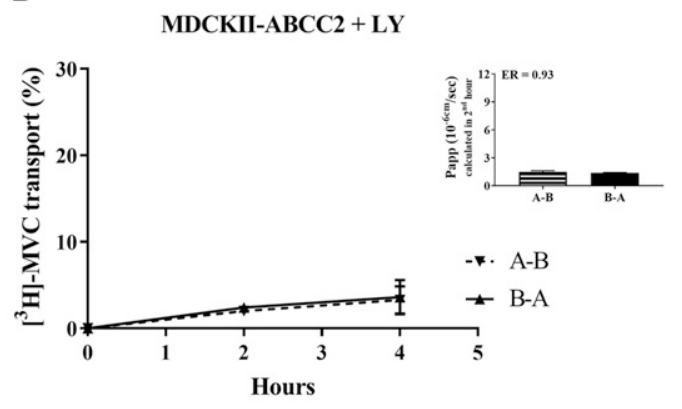

F

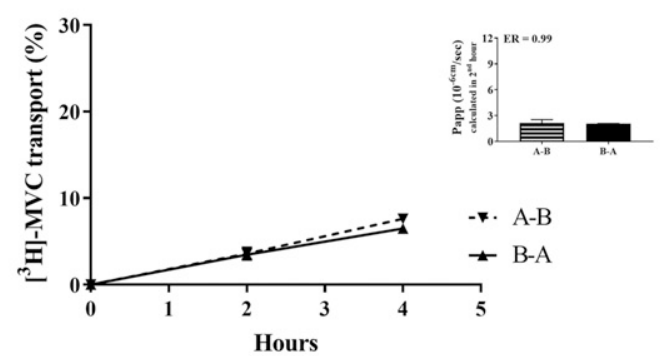

H

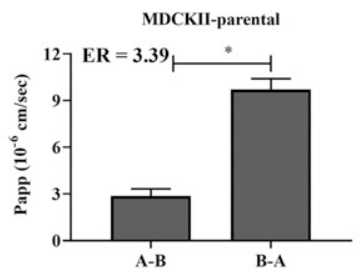

Fig. 2. The bidirectional transport of [ $\left.{ }^{3} \mathrm{H}\right]-\mathrm{MVC}$ across a monolayer of MDCKII-ABCB1 (A and B), MDCKII-ABCG2 (C), MDCKII-ABCC2 (D), MDCKII parental (E and F), and MDCKII-Abcb1 KO cells (G). The recognized ABCB1/Abcb1 substrate digoxin was used to validate the efficiency of endogenous canine Abcb1 KO (H). The transport ratio (ER) was calculated as a ratio of permeability coefficients reached in B-A and A-B direction in the 2nd hour of the experiment. Significantly accelerated transport of $\left[{ }^{3} \mathrm{H}\right]-\mathrm{MVC}$ in the B-A direction was observed in MDCKII-ABCB1 (A) and MDCKII-parental (E) cells, and this dynamic was abolished by ABCB1/Abcb1 inhibitor zosuquidar (LY) addition in both cell lines (B and F). Transport studies in MDCKII-ABCG2 (C) and MDCKII-ABCC2 (D) cell lines were performed in the presence of LY as a background Abcb1 inhibitor. Obtained results in MDCKII-Abcb1 KO cells (G) did not show any transport of $\left[^{3} \mathrm{H}\right]-\mathrm{MVC}$, suggesting the involvement of canine Abcb1 in $\left[{ }^{3} \mathrm{H}\right]-\mathrm{MVC}$ in MDCKII-parental cells (E).

$(10 \mu \mathrm{M})$. No effect was observed after the addition of fumitremorgin $\mathrm{C}(2 \mu \mathrm{M})$, an ABCG2-specific inhibitor.

\section{Discussion}

When administered to pregnant women, maraviroc shows a relatively low fetal-cord/maternal-blood ratio (0.33) (Calcagno et al., 2013;
Colbers et al., 2015). In this study, we hypothesized that the interaction of maraviroc with drug efflux transporters namely, ABCB1, ABCG2, and $\mathrm{ABCC} 2$ may partly explain why this compound, which has relatively high lipophilicity, shows limited transplacental transfer.

The transport of $\left[{ }^{3} \mathrm{H}\right]$-maraviroc across monolayers of MDCKIIABCB1, MDCKII-ABCG2, and MDCKII-ABCC2 cells as well as MDCKII parental cells showed an asymmetry between the B-A and A-B 


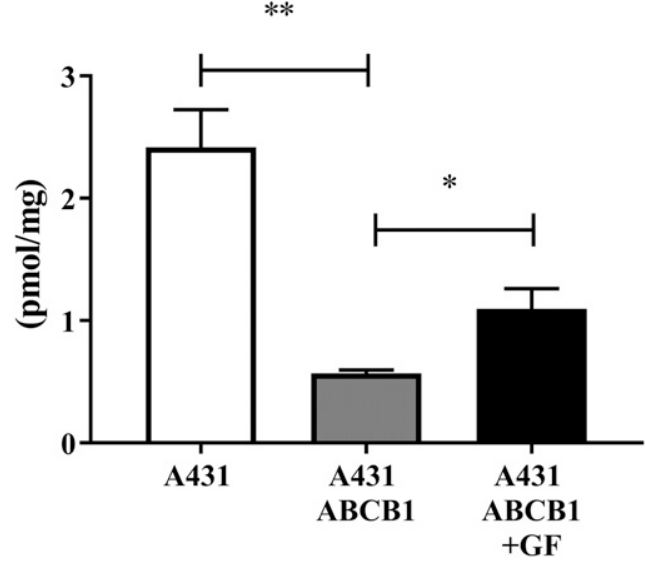

Fig. 3. Accumulation of $\left[{ }^{3} \mathrm{H}\right]-\mathrm{MVC}$ in $\mathrm{A} 431$ and $\mathrm{A} 431-\mathrm{ABCB} 1$ cells. The $\mathrm{A} 431$ cells show noticeably higher $\left[{ }^{3} \mathrm{H}\right]-\mathrm{MVC}$ levels than A431-ABCB1 cells. $\left[{ }^{3} \mathrm{H}\right]-\mathrm{MVC}$ levels increased when the A431-ABCB1 cells were exposed to the ABCB1 inhibitor elacridar (GF), which confirms $\left[{ }^{3} \mathrm{H}\right]-\mathrm{MVC}$ as a substrate of human ABCB1. Data were analyzed by unpaired $t$ test, $* P \leq 0.05 ; * * P \leq 0.01$.

directions, with similar transport efflux ratios of ER $>5$. In all of these cell lines, the asymmetric transport was completely abolished by the addition of the ABCB1/Abcb1 inhibitor zosuquidar. Moreover, no asymmetry was observed in the Abcb1 KO MDCKII-parental cells (ER $=1.08)$, which demonstrates that maraviroc is a substrate of the endogenous canine Abcb1 transporter.

This mechanism has also recently been reported for the novel anticancer drugs ribociclib and dinaciclib (Cihalova et al., 2015; Sorf et al., 2018), which showed efflux ratios higher than 2 in transport assays across MDCKII parental cells. These compounds show strong affinity for human ABCB1, and as such the results from the MDCKII-ABCB1 cells showed even higher ER values, yielding relative ratios between the $\mathrm{ABCB} 1$-overexpressing and parental cells $\left(\mathrm{R}_{\mathrm{r}}=\mathrm{ER}_{\mathrm{ABCB} 1} / \mathrm{ER}_{\text {parental }}\right)$ that were higher than 2 .

According to the International Transporter Consortium guidelines, a relative ratio exceeding the value of 2 confirms that the tested compound is a substrate of the overexpressed human ABC transporters (Giacomini et al., 2010). However, the calculated relative ratio for maraviroc in the present study was $R_{r}=1.20$, which was an unexpected result and forced us to question whether maraviroc is a substrate for human ABCB1.

A similar issue arose in the case of vinblastine. This compound was defined as a dual ABCB1/Abcb1 substrate, yet only a negligible difference was observed when transport assay results from MDCKII parental and MDCKII-ABCB1 cells (Kuteykin-Teplyakov et al., 2010) were compared. Therefore, the expression of canine Abcb1 was evaluated in both cell lines, with the results showing inconsistent Abcb1 mRNA and protein levels.

This imbalance can lead to hiding the real contribution of human $\mathrm{ABCB} 1$ in the transport of substrate with parallel strong affinity to canine Abcb1 (Kuteykin-Teplyakov et al., 2010). Thus, we verified maraviroc transport by human $\mathrm{ABCB} 1$ in resistant $\mathrm{ABCB} 1$-overexpressing human epidermoid A431 cells, showing significantly lower (4.2-fold) intracellular concentrations of the drug than in A431 wild-type cells lacking ABCB1. These results-along with the fact that the ABCB1 inhibitor elacridar partially reversed this effect-provide solid evidence that human $\mathrm{ABCB} 1$ participates in maraviroc transport and has the potential to affect maraviroc pharmacokinetics. However, it is important to consider that these results stem from transport studies in the cocker spaniel kidney MDCK cells; in this way, the canine Abcb1 likely shows higher affinity for maraviroc than human $\mathrm{ABCB} 1$.
To investigate whether the human ABCG2 and ABCC2 transporters influence maraviroc pharmacokinetics, the MDCKII-ABCG2 and MDCKII-ABCC2 cell lines were further exposed to zosuquidar, an inhibitor of Abcb1 not affecting other ABC transporters (Modok et al., 2006). The experiments in MDCKII-ABCG 2 and MDCKII-ABCC 2 cell yielded ER values of 1.42 and 0.93 , respectively, which excluded the possibility that maraviroc is a substrate of either human ABCG2 or ABCC2. Moreover, previous accumulation studies with pheophorbide $A$ in MDCKII-BCRP and calcein-AM in L-MDR1 did not provide evidence that maraviroc inhibits $\mathrm{ABCB} 1$ or $\mathrm{ABCG} 2$ transporters (Zembruski et al., 2011).

We confirmed this lack of interaction using a fluorescent substrate, Hoechst 33342, in MDCKII-ABCG2 and MDCKII-ABCB1 cells, although in the case of $\mathrm{ABCB} 1$, competitive inhibition of maraviroc as an ABCB1 substrate might be expected, in particular at higher concentrations. This discrepancy could be explained by much lower affinity of maraviroc toward human ABCB1 when compared with the high-affinity substrate Hoechst 33342 and/or by the existence of more binding sites on ABCB1 (Subramanian et al., 2019).

Interestingly, while Zembruski et al. (2011) observed an activation of ABCC2 in inside-out membrane vesicles when 5(6)-carboxy$2^{\prime}, 7^{\prime}$-dichlorofluorescein served as the substrate, we were not able to observe this dynamic in accumulation assays in MDCKII-ABCC 2 cells with calcein-AM as the substrate, even when a wide range of maraviroc concentrations (1-50 $\mu \mathrm{M})$ were tested. This suggests possible substratedependent interactions.

Vinot et al. (2013) observed accelerated fetomaternal transfer of maraviroc during an open-circuit perfusion of human placental cotyledon, which suggests that apically located efflux transporter(s) are involved in maraviroc transport. While gene expression profiling of the perfused human placentas suggested that maraviroc may be a substrate of $\mathrm{ABCB} 1$ and $\mathrm{ABCC} 2$, this assumption has never been functionally confirmed in placental tissue with the use of inhibitors. While maraviroc affinity to human ABCB1 was confirmed in our accumulation assays, the transport studies across MDCKII-ABCC2 monolayers did not indicate that the $\mathrm{ABCC} 2$ transporter is involved in the B-A active transport of maraviroc. The dual perfusion of rat term placenta highlighted the enhanced $\mathrm{F} \rightarrow \mathrm{M}$ transplacental passage of maraviroc. This active transport was significantly reduced by several ABCB1 inhibitors-zosuquidar, elacridar, and ritonavir-along with high saturating concentrations of maraviroc. These experiments demonstrated, for the first time, the importance of this interaction in situ.

We conclude that ABCB1 may be partly responsible for the low cord-blood/maternal-blood ratio and accelerated placental transport of

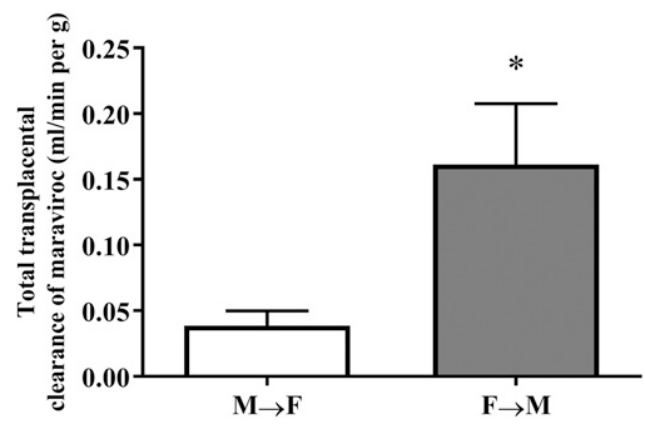

Fig. 4. Transplacental clearance $(\mathrm{M} \rightarrow \mathrm{F}$ and $\mathrm{F} \rightarrow \mathrm{M}$ directions $)$ of $\left[{ }^{3} \mathrm{H}\right]-\mathrm{MVC}$ in open-circuit perfusion of rat term placenta. The significant asymmetry in maraviroc transplacental clearance suggests that an active transport mechanism exists for maraviroc transplacental passage. Columns represent mean \pm S.D. from at least three independent experiments. Data were analyzed by Mann Whitney test, $* P \leq 0.05$. 


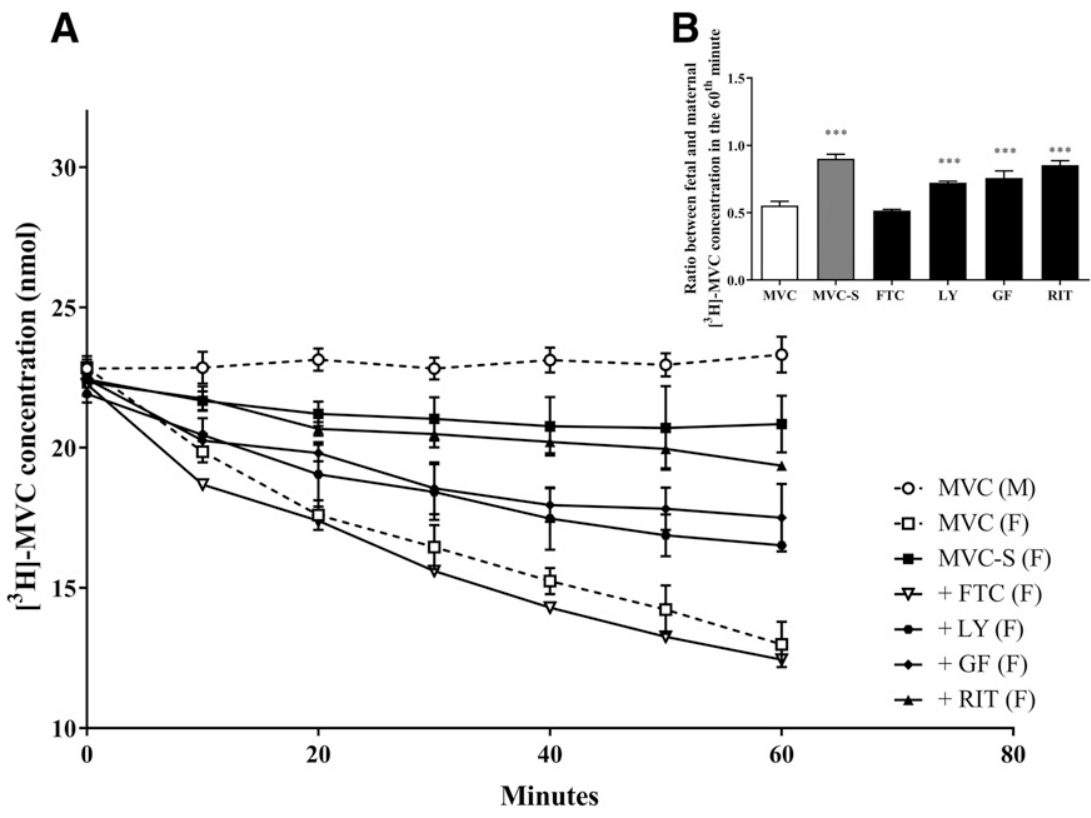

Fig. 5. (A) Decreasing $\left[{ }^{3} \mathrm{H}\right]-\mathrm{MVC}$ concentrations in the fetal compartment during closed-circuit placenta perfusion, shown as MVC (F). The results suggest active maraviroc efflux from the $\mathrm{F} \rightarrow \mathrm{M}$. This transport was reduced in the presence of transporter inhibitors: Abcb1 inhibitor zosuquidar (LY, $2 \mu \mathrm{M})$; dual Abcb1/Abcg2 inhibitor elacridar (GF, $2 \mu \mathrm{M})$; and polyspecific inhibitor ritonavir (RIT, $10 \mu \mathrm{M}$ ). The Abcg2-specific inhibitor fumitremorgin $\mathrm{C}(2 \mu \mathrm{M})$ did not alter $\left[{ }^{3} \mathrm{H}\right]-\mathrm{MVC}$ concentrations in the fetal compartment during the experiments. High concentrations of maraviroc (MVC-S) were used to further verify the saturation and thereby involvement of transport mechanisms. (B) The ratio of concentrations in fetal/maternal compartment was calculated during the last sampling point (60 minutes). Data were analyzed by one-way ANOVA, with Dunnett's multiple comparison test, $* * * P \leq 0.001$

maraviroc in the $\mathrm{F} \rightarrow \mathrm{M}$ direction, both of which were described in humans (Vinot et al., 2013; Colbers et al., 2015). This information could be pivotal to improving the understanding of maraviroc pharmacokinetics and management of combination antiretroviral therapy. Nevertheless, we are also aware that this study is limited by the rat perfusion model. Even though human and rat placentas show significant morphologic and functional similarities, drug affinity to placental ABCB1/Abcb1 may significantly differ across species (de Rijk et al., 2002; Myllynen et al., 2010), and this seems to be the case for maraviroc. Therefore, experiments in human placental tissue are required to verify the role of human $\mathrm{ABCB} 1$ in maraviroc transport across the human placental barrier.

\section{Acknowledgments}

We thank Dana Souckova for her skillful assistance in the perfusion experiments.

\section{Authorship Contributions}

Participated in research design: Ceckova, Tupova, Staud.

Conducted experiments: Tupova, Ambrus, Sorf, Ptackova.

Performed data analysis: Tupova, Ceckova, Sorf.

Wrote or contributed to the writing of the manuscript: Tupova, Ceckova, Gaborick, Sorf, Staud.

\section{References}

Abel S, Russell D, Taylor-Worth RJ, Ridgway CE, and Muirhead GJ (2008a) Effects of CYP3A4 inhibitors on the pharmacokinetics of maraviroc in healthy volunteers. Br J Clin Pharmacol 65 (Suppl 1):27-37.

Abel S, Russell D, Whitlock LA, Ridgway CE, Nedderman AN, and Walker DK (2008b) Assessment of the absorption, metabolism and absolute bioavailability of maraviroc in healthy male subjects. Br J Clin Pharmacol 65 (Suppl 1):60-67.

Calcagno A, Trentini L, Marinaro L, Montrucchio C, D'Avolio A, Ghisetti V, Di Perri G, and Bonora S (2013) Transplacental passage of etravirine and maraviroc in a multidrugexperienced HIV-infected woman failing on darunavir-based HAART in late pregnancy. J Antimicrob Chemother 68:1938-1939.

Ceckova-Novotna M, Pavek P, and Staud F (2006) P-glycoprotein in the placenta: expression, localization, regulation and function. Reprod Toxicol 22:400-410.

Cihalova D, Ceckova M, Kucera R, Klimes J, and Staud F (2015) Dinaciclib, a cyclin-dependen kinase inhibitor, is a substrate of human ABCB1 and ABCG2 and an inhibitor of human ABCC1 in vitro. Biochem Pharmacol 98:465-472.

Colbers A, Best B, Schalkwijk S, Wang J, Stek A, Hidalgo Tenorio C, Hawkins D, Taylor G, Kreitchmann R, Burchett S, et al.; PANNA Network and the IMPAACT 1026 Study Team (2015) Maraviroc pharmacokinetics in HIV-1-infected pregnant women. Clin Infect Dis 61: $1582-1589$. de Rijk EP, van Esch E, and Flik G (2002) Pregnancy dating in the rat: placental morphology and maternal blood parameters. Toxicol Pathol 30:271-282.

Giacomini KM, Huang SM, Tweedie DJ, Benet LZ, Brouwer KL, Chu X, Dahlin A, Evers R, Fischer V, Hillgren KM, et al.; International Transporter Consortium (2010) Membrane transporters in drug development. Nat Rev Drug Discov 9: $215-236$.

Hahnova-Cygalova L, Ceckova M, and Staud F (2011) Fetoprotective activity of breast cancer resistance protein (BCRP, ABCG2): expression and function throughout pregnancy. Drug Metab Rev 43:53-68.

Han LW, Gao C, and Mao Q (2018) An update on expression and function of P-gp/ABCB1 and BCRP/ABCG2 in the placenta and fetus. Expert Opin Drug Metab Toxicol 14 $817-829$

Kuteykin-Teplyakov K, Luna-Tortós C, Ambroziak K, and Löscher W (2010) Differences in the expression of endogenous efflux transporters in MDR1-transfected versus wildtype cell lines affect P-glycoprotein mediated drug transport. $\mathrm{Br} J$ Pharmacol 160: $1453-1463$.

Lu Y, Hendrix CW, and Bumpus NN (2012) Cytochrome P450 3A5 plays a prominent role in the oxidative metabolism of the anti-human immunodeficiency virus drug maraviroc. Drug Metab Dispos 40:2221-2230.

Modok S, Mellor HR, and Callaghan R (2006) Modulation of multidrug resistance efflux pump activity to overcome chemoresistance in cancer. Curr Opin Pharmacol 6 350-354.

Myllynen P, Kummu M, and Sieppi E (2010) ABCB1 and ABCG2 expression in the placenta and fetus: an interspecies comparison. Expert Opin Drug Metab Toxicol 6: $1385-1398$.

Neumanova Z, Cerveny L, Ceckova M, and Staud F (2014) Interactions of tenofovir and tenofovir disoproxil fumarate with drug efflux transporters $\mathrm{ABCB} 1, \mathrm{ABCG} 2$, and $\mathrm{ABCC} 2$; role in transport across the placenta. AIDS 28:9-17.

Office of AIDS Research Advisory Council (OARAC), Panel on Antiretroviral Guidelines for Adults and Adolescents (2019a) Guidelines for the use of antiretroviral agents in adults and adolescents with HIV. http://www.aidsinfo.nih.gov/ContentFiles/ AdultandAdolescentGL.pdf.

Office of AIDS Research Advisory Council (OARAC), Panel on Antiretroviral Therapy and Medical Management of Children Living with HIV (2019b) Guidelines for the use of antiretroviral agents in pediatric HIV infection. http://aidsinfo.nih.gov/contentfiles/lvguidelines/ pediatricguidelines.pdf.

Office of AIDS Research Advisory Council (OARAC), Panel on Treatment of Pregnant Women with HIV Infection and Prevention of Perinatal Transmission (2018) Recommendations for use of antiretroviral drugs in transmission in the United States. http://aidsinfo.nih.gov/contentfiles/ lvguidelines/PerinatalGL.pdf.

Pervaiz A, Ansari S, Berger MR, and Adwan H (2015) CCR5 blockage by maraviroc induces cytotoxic and apoptotic effects in colorectal cancer cells. Med Oncol 32:158

Pervaiz A, Zepp M, Mahmood S, Ali DM, Berger MR, and Adwan H (2019) CCR5 blockage by maraviroc: a potential therapeutic option for metastatic breast cancer. Cell Oncol (Dordr) 42: 93-106.

Sorf A, Hofman J, Kučera R, Staud F, and Ceckova M (2018) Ribociclib shows potential for pharmacokinetic drug-drug interactions being a substrate of ABCB1 and potent inhibitor of ABCB1, ABCG2 and CYP450 isoforms in vitro. Biochem Pharmacol 154 $10-17$.

Staud F, Cerveny L, and Ceckova M (2012) Pharmacotherapy in pregnancy; effect of ABC and SLC transporters on drug transport across the placenta and fetal drug exposure. J Drug Target 20:736-763.

Staud F, Vackova Z, Pospechova K, Pavek P, Ceckova M, Libra A, Cygalova L, Nachtigal P, and Fendrich $Z$ (2006) Expression and transport activity of breast cancer resistance protein (Bcrp/Abcg2) in dually perfused rat placenta and HRP-1 cell line. J Pharmacol Exp Ther 319: 53-62. 
Subramanian N, Schumann-Gillett A, Mark AE, and O'Mara ML (2019) Probing the pharmacological binding sites of P-glycoprotein using umbrella sampling simulations. $J$ Chem Inf Mode 59:2287-2298.

Vinot C, Gavard L, Tréluyer JM, Manceau S, Courbon E, Scherrmann JM, Declèves X, Duro D, Peytavin G, Mandelbrot L, et al. (2013) Placental transfer of maraviroc in an ex vivo human cotyledon perfusion model and influence of $\mathrm{ABC}$ transporter expression. Antimicrob Agents Chemother 57:1415-1420.

Walker DK, Abel S, Comby P, Muirhead GJ, Nedderman AN, and Smith DA (2005) Species differences in the disposition of the CCR5 antagonist, UK-427,857, a new potential treatment for HIV. Drug Metab Dispos 33:587-595.

Woollard SM and Kanmogne GD (2015) Maraviroc: a review of its use in HIV infection and beyond. Drug Des Devel Ther 9:5447-5468.
Zembruski NC, Büchel G, Jödicke L, Herzog M, Haefeli WE, and Weiss J (2011) Potential of novel antiretrovirals to modulate expression and function of drug transporters in vitro. J Antimicrob Chemother 66:802-812.

Address correspondence to: Dr. Frantisek Staud, Department of Pharmacology and Toxicology, Faculty of Pharmacy in Hradec Kralove, Charles University, Akademika Heyrovského 1203, 50005 Hradec Kralove, Czech Republic. E-mail: frantisek.staud@faf.cuni.cz 